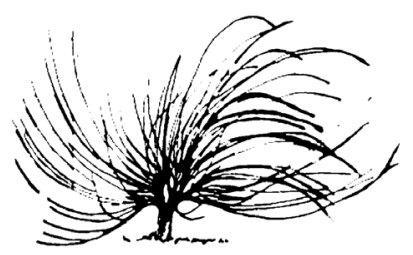

\title{
Entornos virtuales de aprendizaje en comunidades de práctica de docentes universitarios del Ecuador
}

\author{
Zoila Arroyo Vera ${ }^{1}$ \\ Universidad Técnica de Manabí \\ Ecuador \\ zoilaarroyovera@gmail.com \\ Santiago Fernández Prieto ${ }^{2}$ \\ Universidad Autónoma de Madrid \\ España \\ manuel.fernandez@uam.es \\ Lenin Barreto Zambrano ${ }^{3}$ \\ Universidad Técnica de Manabí \\ Ecuador \\ barreto.lenin@gmail.com \\ Luis Ernesto Paz Enrique ${ }^{4}$ \\ Universidad Central "Marta Abreu" de las Villas \\ Cuba \\ luisernestope@uclv.cu
}

\section{(c) (1) $\Theta(\Theta$}

Recibido: 8 de enero de 2018. Aprobado: 24 de setiembre de 2018.

http://dx.doi.org/10.15359/rep.13-2.9

1 Profesor principal de tiempo completo. Máster en Investigación Educativa. Universidad Técnica de Manabí, Ecuador.

2 Director del Departamento de Didáctica de la Educación. Doctor en Ciencias de la Educación. Universidad Autónoma de Madrid, España.

3 Profesor auxiliar de tiempo completo. Máster en Investigación Educativa. Universidad Técnica de Manabí, Ecuador.

4 Profesor Instructor. Licenciado en Ciencias de la Información. Doctorante en Ciencias Sociológicas. Universidad Central "Marta Abreu" de Las Villas, Cuba. 


\title{
Resumen
}

El empleo de los entornos virtuales de aprendizaje supone un cambio de paradigma en las formas en las que se realiza el proceso docente universitario. En Ecuador, existen grandes potencialidades para el uso de entornos virtuales de aprendizaje en la universidad; sin embargo, la mayor parte de los docentes desconoce los beneficios de las plataformas y se rechaza su uso. Se plantearon como objetivos del estudio: 1) examinar aspectos teóricos sobre los entornos virtuales de aprendizaje en el contexto universitario e 2) identificar las características de los entornos virtuales de aprendizaje en el Ecuador. Para la obtención de resultados, se plantearon métodos en los niveles teórico y empírico. Se determinaron las características y beneficios de dichos entornos y se analizaron las ventajas que traería su uso intensivo en la comunidad de práctica de docentes universitarios del Ecuador.

Palabras claves: entornos virtuales de aprendizaje, educación superior, comunidad de práctica educativa, Ecuador.

\begin{abstract}
The use of virtual learning environments represents a paradigm shift in the ways in which the university teaching process is carried out. In Ecuador, there is great potential for the use of virtual learning environments in universities; however, most teachers do not know the benefits of the platforms and refuse to use them. The objectives of the study were: 1) to examine theoretical aspects of virtual learning environments in the university context and 2) to identify the characteristics of virtual learning environments in Ecuador. To obtain results, methods were proposed at the theoretical and empirical levels. The characteristics and benefits of virtual learning environments were discussed and the advantages that their intensive use would bring to the community of teachers' practice in Ecuador were analyzed.
\end{abstract}

Keywords: virtual learning environments, higher education, educational practice community, Ecuador. 


\section{Introducción}

$\mathrm{L}$

as nuevas demandas sociales han desarticulado el paradigma clásico de la educación universitaria tradicional. La universidad como institución social se ha convertido en el instrumento para medir el progreso y el desarrollo. Los sistemas de educación superior debieran aumentar su capacidad para vivir en medio de la incertidumbre, transformarse y provocar el cambio (Paz, Hernándezy Van de Water, 2016).

Una de las características de la sociedad contemporánea es el empleo del conocimiento generado en el seno de las universidades en los procesos productivos. El factor más importante no es ya la disponibilidad de capital, mano de obra, materias primas o energía, sino el uso intensivo del conocimiento y la información (García, 2008).

En América Latina, la dinámica de evolución de las instituciones universitarias ha estado asociada a un conjunto variable de factores en cada momento histórico. La autonomía de las universidades significaba que todo el accionar de estas se basaba en la libertad política y de pensamiento. El poder de autonomía se constituyó como nuevas fuerzas de política dentro y fuera de los países latinos. El modelo de autonomía de las universidades frente al Estado no solo fue el resultado de la redistribución de cuotas de poder. Se concordó en un modelo de desarrollo basado en la sustitución de importaciones, que crecía sin articularse a los centros académicos y produjo la formación de las minorías profesionales (Rikap, 2017). Otro de los aspectos determinantes fueron las reformas universitarias.

A fines de la década de los 60, la lucha por la autonomía tendió a conformar estructuras de poder alternativas. La universidad se consolidó como centro de contrapoder y se perfiló como institución no solo contestataria sino revolucionaria (Aguilera, 2016). El desarrollo de la formación docente universitaria actualmente tiene una tendencia a lo no presencial con el empleo de las tecnologías de la información y las comunicaciones (TIC). El desarrollo de habilidades y competencias tecnológicas constituye una preocupación actual para la docencia universitaria. El uso correcto de las plataformas educativas potencia el intercambio, donde el espacio físico es configurado para una interacción virtual.

Esta modalidad de estudios fue implementada en la Universidad Técnica de Manabí (UTM), Ecuador, en el periodo septiembre-febrero de 2015. Se presentó en la plataforma Moodle, con 8 materias de 
universidad. Se inició con una cantidad de 7000 estudiantes divididos en paralelos de 40 cada uno. Los alumnos pertenecían a todas las facultades de este centro de altos estudios. Transcurrido un período con la modalidad virtual, se han presentado dificultades técnicas o de proveedor de Internet. En el aspecto educativo, las formas de aprendizaje de ciertos estudiantes presentaron complicaciones en el momento de comprender las actividades. Lo anterior se debe al desconocimiento del manejo de la plataforma o de su desenvolvimiento en la utilización de las herramientas asociadas a las TIC.

Los estudiantes del aprendizaje virtual comienzan a experimentar un acercamiento hacia la adquisición de habilidades. Para un desarrollo efectivo dentro del aula virtual, los alumnos deben elevar su conocimiento y no la mera trasferencia de información. Se necesita una buena interacción de los involucrados en el proceso educativo, ya sea en tiempo real, sincrónico o diferido asincrónico. Según Castillo, Ramírez, y Ferrer (2017), hay además, un segundo y crítico aspecto de este entorno más amplio que la conversación y la interacción. No es meramente para crear una red en la que situar aprendizaje episódico, sino para crear una que aprende y, por lo tanto, se adapta y da nueva forma a sí misma, sobre la base de las conversaciones e interacciones.

Los requerimientos del sistema promueven el desarrollo de las habilidades sociales y de cooperación. Son aspectos que fomentan la interacción de estudiante-docente o estudiante-estudiante, según la necesidad o dado el tema que se esté tratando. Las habilidades son promovidas por medio de las actividades que el docente ha establecido en su espacio de aula virtual. A criterio de Espinoza y Rodríguez (2017), los usos pedagógicos en los ambientes de aprendizaje son una respuesta tecnológica. Facilitan la distribución a partir de datos de muy diversa índole, como contenidos y recursos de comunicación propios de Internet.

Los docentes virtuales tendrán la responsabilidad de enriquecer ese espacio de aprendizaje con contenidos claves para el estudiante. El empleo de íconos prácticos y actividades direccionadas a estimular el aprendizaje autónomo generan competencias. Según Pablo (2017), las actividades y su adecuación al aprendizaje son fundamentales en este diseño educativo. Los conceptos de seguimiento y evaluación continuada exigen una continua retroalimentación de un uso más extendido de herramientas como los test, foros, preguntas o exámenes online. 
El término comunidades de práctica constituye una visión contemporánea surgida a finales de la década de 1980. Los autores Lave y Wenger (1991), pioneros en este tipo de investigaciones comunitarias, formularon la primera aproximación teórica:

Una comunidad de práctica se define a sí misma a lo largo de tres dimensiones: su empresa conjunta es comprendida y continuamente renegociada por sus miembros, el compromiso mutuo que se une a sus miembros juntos en una entidad social y el repertorio compartido de recursos comunes (rutinas, sensibilidades, artefactos, vocabulario, estilos) que los miembros han desarrollado a lo largo del tiempo (p. 10).

La conceptualización anterior sustenta que el aprendizaje al interior de la comunidad de práctica es directamente proporcional a la participación de sus integrantes. Las relaciones en esta forma de asociación permiten profundizar en los niveles de identidad grupal y trascienden una simple trasmisión de contenidos e información. Este rasgo distintivo remite a los autores Wing, Pratt, Anderson y Stigter (2006) a sustentar que el proceso de aprendizaje y de pertenencia constituye la esencia para compartir valores, creencias, lenguajes, entre otros.

La búsqueda de opciones efectivas para potenciar el aprendizaje y habilidades en los estudiantes supone el reconocimiento de las bondades de las TIC. El entorno virtual como espacio educativo posee múltiples oportunidades, "una plataforma tecnológica que permita la gestión de su conocimiento" (Redondo, Navarro, Gutiérrez y Iglesias, 2017). Las universidades del Ecuador y la UTM necesitan fuentes que ayuden a potenciar el uso de las plataformas virtuales educativas. Se plantean como objetivos del estudio: 1) examinar aspectos teóricos sobre los entornos virtuales de aprendizaje en el contexto universitario (EVA) e 2) identificar las características de los EVA en el Ecuador.

\section{Materiales y métodos}

El estudio se clasifica como descriptivo con enfoque teórico. Se centra en identificar los beneficios del empleo de EVA de la comunidad de práctica de docentes universitarios del Ecuador y la UTM. Para la obtención de resultados, se emplearon métodos en los niveles teórico y 
empírico. En el nivel teórico, se aplica el inductivo deductivo, analítico sintético, sistémico estructural e histórico lógico. En el nivel empírico, se aplica el análisis documental. Las técnicas aplicadas fueron la revisión de documentos a partir de la consulta de publicaciones seriadas y fuentes especializadas recuperadas, mediante Scirus, Google Académico, EBSCO, Redalyc y SciELO.

\section{Resultados y discusión}

\section{Los EVA en el contexto de la educación superior}

En América Latina, la educación virtual es un fenómeno que se inicia en la década de 1990, después de la introducción de nuevos soportes tecnológicos para el manejo de textos, imágenes y sonido. Esta modalidad se afianza como un servicio telemático que se va desarrollando de manera independiente. Ofrecía diversas operaciones de información y comunicación en Internet. Representó la web, entonces, un nuevo recurso para el desarrollo de la educación.

El empleo de las TIC, en la educación superior, así como la creación de EVA han ido avanzando gradualmente en algunos países de Latinoamérica. La automatización de procesos como la calificación hasta el uso de canales de comunicación como la mensajería o el chat han permitido la interacción docente-estudiante.

En la región, se manifiestan muchos modelos de educación a distancia con educación virtual incorporada. Estos expresan relación entre componentes tecnológicos y componentes docentes. Combinan dinámicas virtuales, semipresenciales o abiertas. Según Guevara (2017), se constata un avance hacia procesos de virtualización asociados a esos modelos semipresenciales que se apoyan en plataformas virtuales de código abierto.

La informatización de la sociedad ha permitido que las modalidades de educación virtual, tanto abierta como a distancia, constituyan una alternativa viable para el ahorro de recursos. En el espacio denominado web 2.0, se presentan diversas alternativas para optimizar el aprendizaje. Las más conocidas son foros, chats, mensajería interna, además de las clases y las actividades o tareas propuestas dentro del aula virtual. En el diseño del entorno virtual, el espacio web ha cambiado dramáticamente estos últimos años; de ser información a ser un lugar de constante aprendizaje e interacción, mezclando culturas, distancias, 
conocimientos. A criterio de Francesc (2009), la web 2.0 y los softwares sociales se han convertido en espacios para la interacción, la comunicación y el intercambio de significados.

El surgimiento de la Internet revolucionó el término educación a distancia, creando un nuevo término: e-learning, también conocido como educación en línea o educación distribuida. Los tres tienen el mismo significado y fundan los espacios denominados entornos virtuales, al aprovechar los recursos de almacenamiento y las nuevas formas de comunicación.

El e-learning se presenta como la facilitación de un aprendizaje, capacitación o programa de educación por medios electrónicos. Implica la utilización de ordenador o dispositivo electrónico para recibir una capacitación o materiales educativos o didácticos (García, 2016). Esta forma de aprendizaje utiliza el paradigma constructivista en la producción y aplicación de las actividades. Estas pueden ser individuales o grupales, siempre de carácter interaccionista. Proponen un aprendizaje colaborativo, siendo el estudiante el actor principal.

Los EVA son espacios educativos alojados en la web. Están conformados por un grupo de herramientas que posibilitan la interacción didáctica en un ambiente electrónico. Posibilitan el acceso desde cualquier punto. Sirven para aplicaciones con contenido didáctico para los docentes y alumnos, la interacción entre estos se puede producir en cualquier momento en tiempo sincrónico o asincrónico.

Los entornos virtuales deben ser el principal espacio de comunicación entre la comunidad virtual que constituye la universidad o institución que se está formando. Estos espacios deben permitir la relación de los estudiantes entre ellos; de los estudiantes con los profesores y de los profesores entre sí, además de cualquier otro integrante de la comunidad universitaria. El alcance de los EVA está diseñado para que el estudiante se acerque al aprendizaje autónomo.

Por consiguiente, el interés se genera mediante el descubrimiento y la necesidad de aprender. A su vez, crea en el estudiante la responsabilidad del cumplimiento de las tareas, las cuales han sido establecidas en un tiempo determinado. La mayoría de los entornos virtuales, tanto propietarios como libres, poseen, en la actualidad, herramientas suficientes como para desarrollar acciones educativas bajo diferentes modalidades. Evolucionan tan rápidamente que los análisis de sus principales características son cambiantes. 
Sobre los EVA, el autor Flores (2012) los refiere como la capacitación no presencial a través de plataformas tecnológicas. Posibilitan y flexibilizan el acceso y el tiempo en el proceso de enseñanza-aprendizaje. Se adecúan a las habilidades, necesidades y disponibilidades de cada discente. Garantizan ambientes de aprendizaje colaborativos, mediante el uso de herramientas de comunicación sincrónica y asincrónica. Potencian, en suma, el proceso de gestión basado en competencias. Es un cambio de actitud docente hacia esta nueva comunicación que se presenta en las aulas virtuales.

El docente está comprometido a seguir renovándose en los últimos adelantos en el manejo o diseño de las aulas virtuales. Estas deben presentar un modelo estéticamente pedagógico y funcional, mostrando canales de comunicación atractivos y accesibles. Lo anterior favorecerá la obtención de mejores resultados en la interacción con los estudiantes.

Los nuevos roles en la educación universitaria transitan por términos que se asocian a las TIC. La nueva generación nacida inmersa en el mundo cibernético se denomina "nativos digitales". Rodríguez (2008) afirma que son niños y jóvenes nacidos después de la década de 1990, para quienes lo digital es ya parte de sus vidas. Nacieron y crecieron entre pantallas e Internet. No obstante, el estrato social o cultural de procedencia, el primer contacto con las tecnologías digitales ya los predispone a su apropiación. Las aprenden más rápido que los inmigrantes digitales.

Se considera la alta probabilidad de que los cerebros de los nativos digitales sean fisiológicamente distintos de los de los inmigrantes, como consecuencia de los estímulos digitales que han recibido a lo largo de su crecimiento (Premsky, 2010). Marc Prensky denomina a las generaciones que no crecieron con las nuevas tecnologías inmigrantes digitales. Son aquellas personas que les ha tocado adaptarse a los nuevos cambios. Aprenden lentamente, cada uno a su ritmo, pero sin dejar del todo su pasado. Les toca adquirir un nuevo lenguaje para comunicarse con sus hijos, quienes son expertos y se han adaptado al contexto en que nacieron.

A los nativos digitales les gusta compartir y distribuir el máximo de la información que reciben de forma rápida. Difundir experiencias, estados de ánimo, actitudes y emociones forma parte de la vida cotidiana de los internautas. Es una generación que ha nacido y se ha desarrollado en tiempos de Internet, que cuida su identidad digital y adopta una actitud diferente ante las cosas (en términos de compartir, crear, comunicar, coordinar y aprender). Escofet, López, y Álvarez (2014) han resumido tanto la postura 
optimista como la más crítica sobre los nativos digitales y sus competencias en relación con el uso en ámbitos académicos, especialmente en el universitario. Los autores mencionados concluyen, sobre los nativos digitales, que los estudiantes nacidos en la era de las nuevas tecnologías pueden tener "una mejor disposición para manipular las nuevas tecnologías en el ámbito social [pero]... no se puede concluir de esto que será igualmente hábiles para los usos académicos de las TIC" (p. 19).

Los jóvenes de la nueva época han aprendido a utilizar mejor los aparatos que sus propios padres y maestros. Van un paso adelante con el desarrollo de las tecnologías en comparación con sus antecesores y están reconfigurando la forma de ver el mundo (Madrigal, Madrigal y Gallegos, 2016). Estas personas, naturalmente, presentan habilidades para desenvolverse con las nuevas tecnologías y no necesitan formarse para adquirirla (Burin, Coccimiglio, González y Bulla, 2016). En la actualidad, la parte educativa presenta uno de los mayores retos en cuanto a la alfabetización digital, mediante herramientas informáticas como el ordenador, software, Internet, entre otros. No solo es el saber utilizarlos, sino que se debe lograr el buen entendimiento de los programas codificados, permitiendo así que los individuos desarrollen una nueva forma de adquirir conocimientos.

Los autores Avello, López, Cañedo, Álvarez, Granados y Obando (2013) afirman que los escenarios tecnológicos actuales donde se desarrollan los procesos educativos tienen que ser digitales y multimediáticos. Además, deben considerarse las trasformaciones sociales provocadas por las nuevas herramientas de la web 2.0. Las personas pueden interactuar, crear y compartir una gran variedad de contenidos usando múltiples medios: sonoros, visuales, hipertextuales, simuladores, juegos y redes sociales.

Los profesores en los EVA, en su mayoría, serían "inmigrantes digitales"; sin embargo, los estudiantes son "nativos". Lejos de que lo anterior fuera una limitación, la construcción colectiva de conocimientos constituye uno de los principios fundamentales de los EVA. Estudiantes y profesores compartirían sus experiencias en beneficio mutuo. Se deben sugerir, por tanto, la conformación de grupos cooperativos entre estudiantes, profesores o ambos. El estudiante asume, con este paradigma, un nuevo papel transformador en sus procesos de aprendizaje virtual. Las plataformas virtuales permiten gestionar, administrar e impartir cursos en línea. Ofrecen una serie de contenidos con 
actividades que facultan la comunicación e interacción de los participantes. Es importante que los contenidos sean aplicables y concuerden con las tareas propuestas en el aula virtual.

\section{Los EVA en el Ecuador}

La historia de la educación a distancia en el Ecuador tiene sus orígenes en 1962 con la fundación de las Escuelas Radiofónicas Populares. Esta experiencia se constituye a partir de organizaciones sin fines de lucro, creadas para brindar educación a los sectores populares del campo, de la ciudad e indígenas. En 1974, se funda el Instituto Radiofónico Fe y Alegría, dirigido a niños, padres y madres de familia. Este sistema fue abordado mediante la modalidad distancia presencial y empleó la enseñanza por medio de la radio. Otros autores afirman que la experiencia no fue consolidada hasta 1976 en la Universidad Técnica Particular de Loja, donde se crea la Modalidad Abierta para democratizar la enseñanza universitaria (Álvarez, Bartra, López y Sandovalet, 2015).

El artículo 43 del Reglamento del Régimen Académico del Ecuador autoriza que el componente de docencia, prácticas de los aprendizajes y la parte autónoma estén medidos por el uso de tecnologías y entornos virtuales (Rea y Medina, 2016). En el Ecuador, se han desarrollado experiencias de programas a distancia y semipresenciales con apoyo de Internet, desde el 2001. Desde el 2009, la educación superior en el Ecuador ha experimentado profundos cambios y nuevas exigencias. Contempla que los programas podrán ser en modalidad de estudios presencial, semipresencial, a distancia virtual, en línea y otros. El aprendizaje es favorecido por el uso de tecnologías informáticas y entornos virtuales que consolidan la interacción entre profesores y estudiantes, sin las restricciones del tiempo que suceden en la modalidad presencial directa.

El soporte tecnológico más empleado para la creación de entornos virtuales de aprendizaje se denomina Moodle. El software permite el desarrollo de sistemas de gestión de la enseñanza y favorece el diseño de cursos en línea interactivos (Zúñiga y Martínez, 2015). Los sistemas e-learning también se denominan Sistema de Gestión de Aprendizaje o Ambientes Virtuales de Aprendizaje. El uso masivo de la plataforma Moodle ha permitido consolidar la experiencia de sus usuarios como una herramienta capaz de potenciar el aprendizaje presencial, en línea o una combinación de ambos. 
La comunidad de usuarios y desarrolladores de la plataforma Moodle es numerosa y se caracteriza por su entusiasmo respecto al sistema. Puede afirmarse que es un proyecto inspirado en la pedagogía del constructivismo social y orientado a favorecer habilidades en torno al autoaprendizaje. Es un sistema muy flexible que funciona prácticamente en cualquier plataforma; muy fácil de administrar y operar González (2007).

El software libre aplicado a contextos educativos favorece el proceso de enseñanza-aprendizaje en función de los destinatarios. La docencia vinculada con Moodle se traduce en altos niveles de interactividad por medio de mensajería, chats, foros, anuncios, entre otros. Las clases están numeradas, basándose en el programa de enseñanza-aprendizaje, el cual está organizado por fechas, según el requerimiento de la universidad y del docente. La principal limitación que se identifica para el empleo de la educación virtual la constituye la ausencia de una estructura básica en la conexión a Internet.

El empleo de los cursos virtuales en las instituciones educativas puede incrementar la oferta de programas de estudio inclusivos que favorezcan altos niveles de superación profesional en la sociedad. Otra de las facilidades de estas modalidades on-line la conforman el acceso permanente a todas las fuentes de información. Desde el punto de vista didáctico, una de las innovaciones más profundas se manifiesta en la posibilidad de los estudiantes para la selección de los contenidos que desean consultar, lo que permite superar el modelo expositivo que se evidenciaba en el sistema tradicional de enseñanza. El reto pedagógico constituye la alfabetización de los estudiantes, pues el profesor debe dejar de ser un transmisor de información para convertirse en un tutor que guía y supervisa el proceso del alumnado (Salinas, 2004).

Otro beneficio de la educación virtual radica en el fomento de habilidades para solucionar problemáticas con alto rigor profesional, a partir del trabajo independiente. Se incrementa la capacidad de decidir, con base en sus propios intereses, los conocimientos que el estudiante necesite. La capacidad de seleccionar su currículo formativo constituye una idea valiosa que hace flexible el conocimiento, lo cual permite, según Martínez, Mauri, Colomina, Agirre, Marc, Bilbatua, Onrubia y Pablo (2017), "mejorar su experiencia práctica y representar reflexivamente su futura labor docente" ( $p$. 47). El educador se convierte en facilitador, para que el estudiante avance a su ritmo y con sus propios intereses y necesidades. 
Entre las limitaciones que se presentan actualmente en la enseñanza virtual, se puede señalar que existen muchas desigualdades respecto a la accesibilidad de Internet, bajos niveles de alfabetización informacional sobre los entornos virtuales, resistencia al cambio por parte de grupos sociales que no son considerados nativos digitales e inexistencia, en algunos casos, de la infraestructura necesaria para la implementación de esta tecnología.

Un aspecto imprescindible de la virtualización lo compone la interacción usuario-sistema, lo que exige de las universidades tradicionales un mayor nivel de interactividad con sus usuarios en el mundo virtual. Resulta necesario un cambio que favorezca el tránsito desde la interactividad informativa hacia la interactividad transaccional. Los entornos virtuales universitarios deben poseer un diseño más atractivo que permita integrarse a la cultura de la organización. El usuario es, sin duda, el centro de todo proceso de generalización de una nueva tecnología y la garantía de su éxito.

Los entornos virtuales de aprendizaje han cambiado el paradigma educativo basado en la forma bancaria (Freire, 1970). De este modo, trascienden el esquema clásico de comunicación emisor-receptor, al situar en el proceso elementos tales como la interactividad e hipertextualidad. La relación modalidad de docencia/horario docente no constituye una limitación, pues desde ambos roles (estudiante/profesor) se establecen relaciones propicias para la colaboración. La interacción didáctica que se desarrolla favorece un marco multirelacional, configurado entre docentes y estudiantes (Londoño, Calderón, Lucumí y González, 2017).

Dentro del EVA de la UTM, hay 33 docentes que están trabajando bajo esta modalidad, los cuales han formado una comunidad de interacción, en la que se evidencian los alcances y limitaciones anteriormente expuestos. Los docentes virtuales de la universidad constituyen una muestra piloto, ya que esta modalidad se implementó a partir del 2015. El trabajo metodológico de los docentes en talleres y sesiones plenarias permite proyectar estrategias para el proceso de educación y aprendizaje, así como vías para generalizar los espacios colaborativos. La aceptación de los estudiantes hacia la modalidad virtual se refleja en el correcto funcionamiento de la plataforma y en la participación de estos en las distintas herramientas de la web 2.0.

La comunidad de práctica en entornos virtuales educativos se entiende como un colectivo de docentes que comparte una visión de la enseñanza y elige el debate continuado y público de ideas como un 
vehículo para mejorar su práctica y desarrollarse profesionalmente (González, 2007). Dentro de esta comunidad, se efectúa asignación de roles y responsabilidades, de acuerdo con las asignaturas y metodologías propias. Pero el resultado no se constata de forma individual, ya que las soluciones se complementan entre sí, logrando ser aprovechadas para generar nuevos conocimientos, los cuales son implementados de forma colectiva e inciden en el ambiente de trabajo del docente.

\section{Conclusiones}

Los entornos virtuales de aprendizaje facilitan los procesos pedagógicos y académicos. El constante desarrollo tecnológico de estos espacios necesita personal de soporte capacitado para administrar tal infraestructura. La utilización de esta modalidad debe generar planes de implementación que contemplen soluciones eficientes ante errores informáticos, problemas de conectividad y desconfiguraciones. Previos a la implementación, se requieren procesos de alfabetización mediática e informacional, que favorezcan la socialización entre estudiantes y profesores. El correcto funcionamiento de estos entornos posibilita la creación de comunidades de práctica virtual. La inadecuada puesta en práctica de estos espacios puede generar rechazo por parte de los usuarios potenciales. La baja velocidad de conexión a los espacios virtuales o el déficit de procesamiento de la información, por parte del sistema, puede provocar conflictos en el proceso docente-educativo.

El soporte tecnológico más empleado para la creación de entornos virtuales de aprendizaje en Ecuador se denomina Moodle. Los EVA han favorecido la creación de comunidades de práctica entre los docentes universitarios, pero aún se desconocen las potencialidades de estas herramientas para desarrollar la educación virtual efectiva; aspecto que actúa en detrimento de esta modalidad educativa, que hace uso intensivo de las tecnologías de la información y la comunicación. Fomentar el empleo de los EVA en los centros educativos del Ecuador se revertiría en la calidad de los procesos formativos, a partir de las características de los estudiantes que son, en su mayoría, nativos digitales. Esta tipología de usuario necesita procesos formativos que estimulen el uso de las tecnologías, para fomentar la aprehensión de los conocimientos. A partir de la identificación de las características de los EVA en Ecuador, descritas anteriormente, se cumplen los objetivos de esta investigación. 


\section{Referencias}

Aguilera, A. (2016). Autonomía universitaria: asunto público de interés privado. Revista Colombiana de Educación, 70, 125-148. Recuperado de https://www.scielo.org.co/pdf/rcde/n70/n70a07.pdf

Álvarez, F., Bartra, F., López, E. y Sandoval, Y. (2015). Experiencias universitarias en escenarios virtuales formativos. Cali: Editorial USC.

Avello, R., López, R., Cañedo, M., Álvarez, H., Granados, J. y Obando, F. (2013). Evolución de la alfabetización digital: nuevos conceptos y nuevas alfabetizaciones. Medisur, 11, 450-456. Recuperado de https://scielo.sld.cu/scielo. php?script=sci_arttext\&pid=S1727-897X2013000400009

Burin, D., Coccimiglio, Y., González, F. y Bulla, J. (2016). Desarrollos recientes sobre Habilidades Digitales y Comprensión Lectora en Entornos Digitales. Psicología, Conocimiento y Sociedad, 6, 191-206. Recuperado de https://www.scielo.edu.uy/pdf/pcs/ v6n1/v6n1a09.pdf

Castillo, A., Ramírez, M. y Ferrer, R. (2017). Aula virtual como estrategia para el aprendizaje de la Química Orgánica.Educ@ción en Contexto, II(5), 95-111. Recuperado de https://dialnet.unirioja. es/descarga/articulo/6296651.pdf

Escofet, A., López, M. y Álvarez, G. (2014). Una mirada crítica sobre los nativos digitales: Análisis de los usos formales de tic entre estudiantes universitarios. Revista Q, 9, 1-19. Recuperado de https:// revistas.upb.edu.co/index.php/revista_Q/article/view/7714/0

Espinoza, L. A. y Rodríguez, R. (2017). La generación de ambientes de aprendizaje: un análisis de la percepción juvenil. Revista Iberoamericana para la Investigación y el Desarrollo Educativo, 7(14), 1-19. Recuperado de https://www.ride.org.mx/index.php/ RIDE/article/view/276/1305

Flores, K. M., Bravo, M. S. (2012). Metodología PACIE en los ambientes virtuales de aprendizaje para el logro de un aprendizaje colaborativo. Revista Electrónica Diálogos Educativos, 12(24), 3-17. Recuperado de https://dialnet.unirioja.es/servlet/ articulo? codigo $=4156135$

Francesc, E. (2009). Bolonia y las TIC: de la docencia 1.0 al aprendizaje 2.0. La Cuestión Universitaria, 5, 58-67. Recuperado de 
https://polired.upm.es/index.php/lacuestionuniversitaria/article/ viewFile/3337/3402

Freire, P. (1970). Pedagogía del oprimido. Montevideo: Editorial Tierra Nueva.

García, C. y Cabero, J. (2016). Evolución y estado actual del e-learning en la formación profesional española. RIED. Revista Iberoamericana de Educación a Distancia, 12(2), 167-191. Recuperado de http://revistas.uned.es/index.php/ried/article/view/15800

García, J., Castillo, A. y Aguilera, J. (2008). Sociedad del conocimiento y políticas neoliberales: la escuela bajo acoso. Revista Latinoamericana de Estudios Educativos, 38(1), 35-59. Recuperado de https://www.redalyc.org/articulo.oa?id=27012437003

González, R. A. (2007). La investigación en la práctica educativa: Guía metodológica de investigación para el diagnóstico y evaluación en los centros docentes (No. 5). Madrid: Ministerio de Educación y Ciencia.

Guevara, E., Pardo, M. M. e Izquierdo, J. M. (2017). Reflexión acerca de la virtualización académica universitaria en la Universidad Estatal de Bolívar en Ecuador. Revista Didasc@lia, 8(7), 1-7. Recuperado de https://runachayecuador.com/refcale/index.php/ didascalia/article/view/1864

Lave, J. y Wenger, E. (1991). Situated learning: Legitimate peripheral participation. Cambridge: Cambridge University Press.

Londoño, O. L., Calderón, L. C., Lucumí, P. y González, M. A. (2017). Experiencia innovadora de la estructuración ontológica del conocimiento con docentes y estudiantes. Praxis y Saber, 8(16), 83-104. Recuperado de https://www.scielo.org.co/pdf/prasa/ v8n16/2216-0159-prasa-8-16-00083.pdf

Madrigal, S., Madrigal, F. y Gallegos, A. E. (2016). E-marketing para los dispositivos móviles: los nativos digitales y la cuarta panta1la. Revista de la Facultad de Contaduría y Ciencias Administrativas, 1, 296-311. Recuperado de https://ideas.repec.org/a/msn/ rfjrnl/v1y2016ilp296-311.html

Martínez, A., Mauri, T., Colomina, R., Agirre, N., Marc, C., Bilbatua, M., Onrubia, J. y Pablo, G. (2017). Factores que favorecen la presencia docente en entornos virtuales de aprendizaje. Tendencias Pedagógicas, 3, 43-58. Recuperado de https://revistas.uam. es/tendenciaspedagogicas/article/view/7083 
Paz, L. E., Hernández, E. A. y Van de Water, H. (2016). Los retos de la Educación Superior en el Siglo XXI. Revista Conrado, 12(55), 17-24. Recuperado de https://conrado.ucf.edu.cu/index.php/ conrado/article/download/343/338/

Premsky, M. (2010). Nativos e Inmigrantes Digitales. Madrid: Institución Educativa SEK.

Rea, M. y Medina, J. (2016). La educación a distancia como mecanismo de inclusión social en las universidades del Ecuador. Alternativas, 17(2), 5-10. Recuperado de https://editorial.ucsg.edu.ec/ ojs-alternativas/index.php/alternativas-ucsg/article/view/113

Redondo, S., Navarro, E., Gutiérrez, S. e Iglesias, I. (2017). Mejora del aprendizaje en las organizaciones a través de comunidades virtuales. Revista Complutense de Educación, 28(1), 101-123. Recuperado de https://revistas.ucm.es/index.php/RCED/article/ view $/ 48973$

Rikap, C. (2017). Percepciones sobre la autonomía universitaria de los docentes-investigadores de la Facultad de Farmacia y Bioquímica de la Universidad de Buenos Aires. Revista Iberoamericana de Educación Superior, 8(23), 138-162. Recuperado de https:// www.scielo.org.mx/pdf/ries/v8n23/2007-2872-ries-8-23-00138. pdf

Rodríguez, D. (2008). Nuevas tecnologías Web 2.0: Hacia una real democratización de la información y el conocimiento. Recuperado de http://eprints.rclis.org/11814/1/Rodriguez-DianaTRABAJOelis.pdf

Salinas, J. (2004). Innovación docente y uso de las TIC en la enseñanza universitaria. Revista de Universidad y Sociedad del Conocimiento (RUSC), 1(1), 1-16. Recuperado de https://dialnet.unirioja.es/descarga/articulo/1037290.pdf

Wing, K., Pratt, K., Anderson, M. y Stigter, J. (2006). Literature Review and Synthesis: online communities of practice. A report submitted to the Ministry of Education. Dunedin: University of Otago.

Zúñiga, M. y Martínez, R. (2015). Los entornos virtuales: una necesidad en el mejoramiento de la dinámica del proceso de enseñanza-aprendizaje del Curso Presencial Intensivo en el ITB de Guayaquil. Revista Santiago, 3, 120-144. Recuperado de https:// revistas.uo.edu.cu/index.php/stgo/article/view/729 